\title{
Soft UV Nanoimprint Lithography: A Tool to Design Plasmonic Nanobiosensors
}

\author{
Grégory Barbillon \\ Laboratoire Charles Fabry de l'Institut d'Optique - CNRS UMR 8501, \\ Institut d'Optique Graduate School, \\ France
}

\section{Introduction}

The capability for realizing high density nanostructures over large areas is important for the sensing of chemical and biological molecules based on localized surface plasmon resonance (LSPR) of metallic nanoparticles (Jensen et al., 1999; Barbillon et al., 2008; Faure et al., 2008). To characterize these plasmonic nanosensors on an area of $\sim 100 \times 100 \mu \mathrm{m}^{2}$ (Barbillon et al., 2009; Anker et al., 2008; Barbillon et al., 2008), extinction spectroscopy measurements are mainly used. In order to study multiple biomolecular interactions on the same surface, very large areas need to be fabricated. Various techniques such as focused ion beam lithography and electron beam lithography are available to design these large surfaces. However, these two techniques are slow to obtain these surfaces. Moreover, charge effect on insulating surface can alter the regularity of the pattern shape. Thus, these techniques will not be suitable for a large scale production. Other lithographic techniques such as extreme UV lithography are also used, but these techniques (fabrication of masks) are expensive and allow with difficulty to realize samples in small quantity. In addition, alternative methods emerged, and among these methods we find soft UV nanoimprint lithography (UV-NIL). The UV-NIL process is fast to realize high density nanostructures, not very expensive and compatible with biological and biochemical applications (Krauss \& Chou, 1997). With UV-NIL, samples can be fabricated at room temperature and low pressure. A limiting factor of UV-NIL exists and this factor is the resolution of the fabricated molds (Jung et al., 2006; Austin et al., 2005). Flexible molds of the soft UV-NIL technique were fabricated by cast molding processes, in which an appropriate liquid mold material is deposited on a patterned master mold, followed by optical curing of the material. Moreover, a great homogeneity of patterns is obtained with soft UV-NIL on a large zone. Thus, the purpose of this chapter is to present in details the principle of soft UV-NIL and the results of plasmonic structure fabrication on glass substrates obtained by this technique in order to realize LSPR nanosensors for biological molecules. To finish, a plasmonic sensing of biomolecules is investigated in order to validate the use of soft UV-NIL.

\section{Soft UV nanimprint lithography: Principle \& steps of fabrication}

\subsection{Principle of UV-NIL}

The principle of soft UV-NIL is illustrated in figure 1 and consists of a UV transparent mold that is used to imprint the desired pattern in the UV sensitive resist. This UV sensitive resist, which is liquid at room temperature, is spin coated on the substrate. The UV transparent 
stamp is deposited on the substrate with a low pressure between 0 and 1 bar (Hamouda et al., 2009), at room temperature. Cross-linking of the UV sensitive resist is then performed by exposing the sample, for example, to a UV lamp source (Hamamatsu LC8 at $365 \mathrm{~nm}$ ) ensuring a dose of around $10^{5} \mathrm{~J} / \mathrm{m}^{2}$ (Hamouda et al., 2010). Next, the soft stamp is released, leaving the UV sensitive resist patterned. The removal of residual layer of UV sensitive resist is realized by etching using Reactive Ion Etching (RIE) in order to obtain the desired patterns. The technique of soft UV-NIL has several advantages like 3D structure generation, and the fabrication of patterns on non-planar surfaces. Indeed, the main advantages of this technique are the transparent flexible stamp and a low viscosity UV-curable resist. These flexible stamps are typically replicated by molding and curing a polymer from a 3D template. The most used materials for UV transparent flexible stamp fabrication is poly(dimethylsiloxane) PDMS (Barbillon et al., 2010), which exhibits interesting properties like good chemical stability and high optical transparency. The fabrication process of soft UV-NIL is divided (figure 1) in 6 steps: (1) the master mold fabrication, which allows flexible stamp realization, (2) the flexible stamp fabrication, (3) the substrate is coated with a UV-curable resist layer, (4) the soft PDMS stamp deposition on the substrate with a low pressure, (5) curing of the photoresist with UV illumination through the transparent stamp, (6) demolding of the soft stamp. Moreover, the use of low viscosity UV-curable resists allows 3D patterning at low pressure without any heating cycles, and thus, the deformation risk of the soft stamp is minimized.

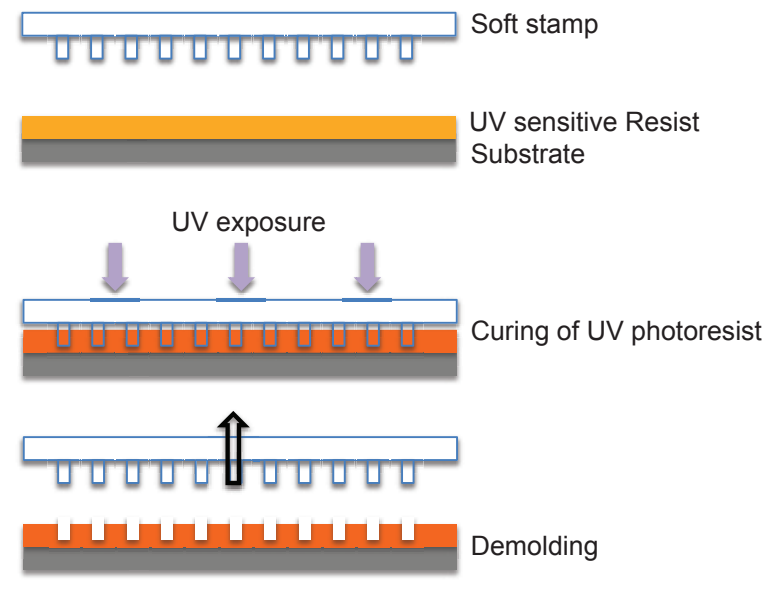

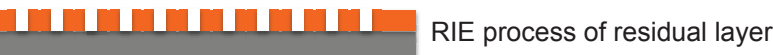

Fig. 1. Principal steps of UV nanoimprint lithography.

\subsection{First step of fabrication: Master mold}

The method, which is mainly used to fabricate high resolution of nanostructures, is the electron beam lithography (EBL). The advantages of EBL are great accuracy, a very high resolution, and an ability to pattern a large variety of geometries. In the example presented here for Si master mold fabrication, an EBL system (Raith 150) is used to expose the PolyMethylMethAcrylate A6 resist (PMMA A6), employing an accelerating voltage of $20 \mathrm{kV}$, 
aperture $7.5 \mu \mathrm{m}$ and working distance of $7 \mathrm{~mm}$. Next, the patterns designed in PMMA are transferred into the silicon master via a suitable RIE process. The conditions of RIE process are: $10 \mathrm{sccm}$ for $\mathrm{O}_{2}, 45 \mathrm{sccm}$ for $\mathrm{SF}_{6}$ with $\mathrm{P}=30 \mathrm{~W}$, a pressure of $50 \mathrm{mTorr}$ and an autopolarization voltage of $85 \mathrm{~V}$ (Hamouda et al., 2009; Barbillon et al., 2010). Then, the PMMA mask is removed with acetone. After these steps, the Si master mold surface is treated with $\mathrm{HF}$ and $\mathrm{H}_{2} \mathrm{O}_{2}$ to get a $\mathrm{SiO}_{2}$ thin surface layer, then modified with an anti-sticking layer (TMCS: TriMethylChloroSilane) to lower the surface energy ( $\mathrm{Si}+\mathrm{TMCS}=28.9 \mathrm{mN} / \mathrm{m}$ ) which eases the removal of the PDMS molds. In figure 2, SEM images of obtained nanostructures are shown. The chosen geometry for biochemical sensing is nanodisk and consequently, the pattern geometry for the Si master mold designed by EBL is nanohole with following dimensions: diameter of $80 \mathrm{~nm}$ and a periodicity of $250 \mathrm{~nm}$.

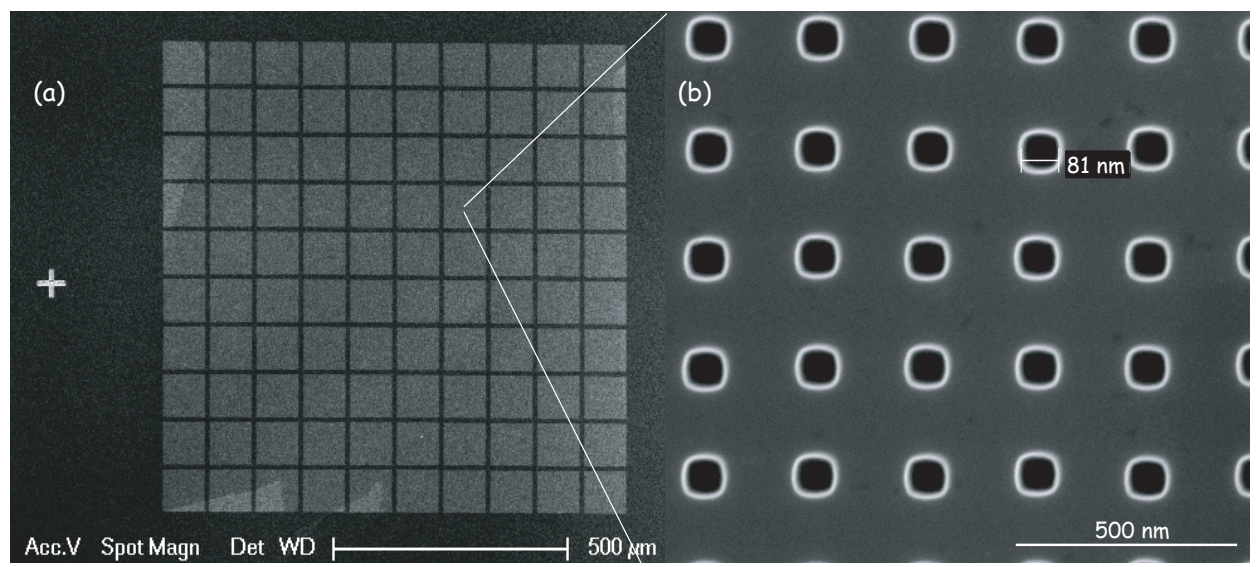

Fig. 2. SEM images of Si master mold designed by EBL: nanoholes of diameter $80 \mathrm{~nm}$ and 250 $\mathrm{nm}$ of periodicity on a zone of $1 \mathrm{~mm}^{2}$. (a) the zone of $1 \mathrm{~mm}^{2}$ and (b) zoom of one square zone where are the nanoholes.

In addition, we developed an alternative method with EBL in order to realize the master mold. This technique is that of nanoporous anodic aluminum oxide (AAO). Nanoporous alumina substrates exhibit an arrangement of nanometric pores, organized in a hexagonal lattice on very large surfaces $\left(\mathrm{Ex}\right.$ : some $\mathrm{cm}^{2}$ ). The dimensions of vertical pores can be easily tuned as the diameter, the aspect ratio. The magnitude order of diameter is of 10 to $200 \mathrm{~nm}$, and the aspect ratio can be higher than 500. The synthesis of AAO is realized electrochemically from aluminum wafers. After a step of polishing, anodic potential is applied to an aluminum wafer, at a given and controlled temperature, immersed in an acid bath. The key parameter is the anodization voltage for the growth of these highly ordered nanoporous membranes. In the example that we present here, experimental conditions are chosen in order to obtain membranes with thicknesses of around $10 \mu \mathrm{m}$ and holes diameter of $180 \mathrm{~nm}$ (see figure 3) (Sengupta et al., 2009; Masuda \& Fukuda, 1995). Thus, metallic nanodisks will be obtained for plasmonic biosensors application (not shown for this case in this chapter) by using this technique of AAO templates for the UV-sensitive flexible stamp fabrication (Hamouda et al., 2011). 


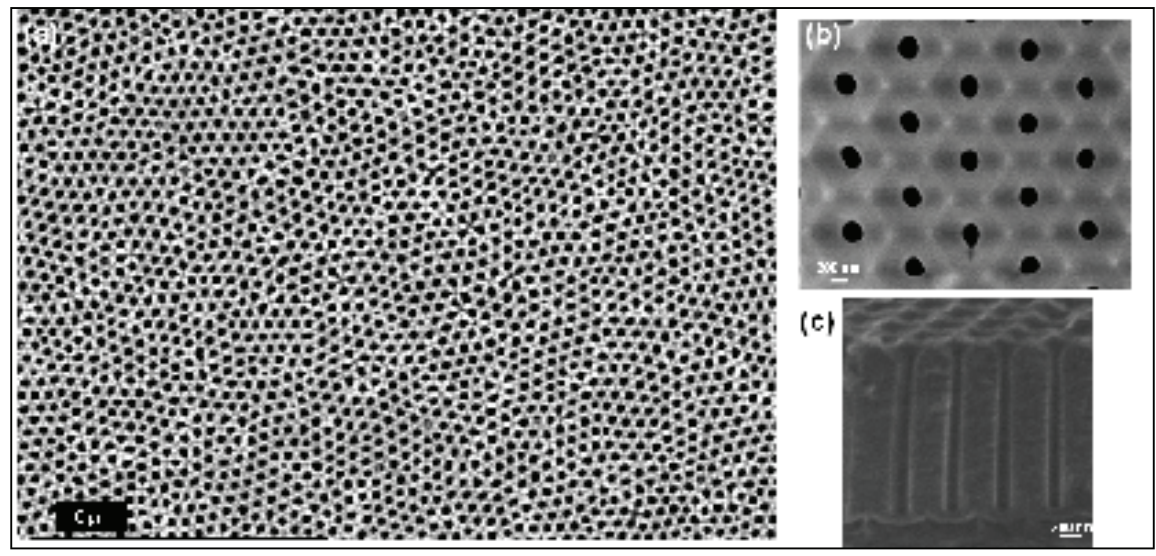

Fig. 3. SEM images of AAO templates: (a) Large view of nanopores, (b) Zoom of these nanopores and (c) Height profile of these vertical nanopores.

\subsection{Second step of fabrication: Flexible UV-transparent stamp}

For the fabrication of the soft UV-transparent stamp, an elastomer is used on which are realized the desired patterns. The material most used in UV-NIL is poly(dimethylsiloxane) and this material has attractive properties like its low Young's modulus (Bender et al., 2004), its low surface energy, which allows conformal contact with surface without applied pressure and nondestructive release from designed structures (Hsia et al., 2005) and its good transparency to a UV light source (Schmid et al., 1998). Mainly, the used material was undiluted PDMS (RTV 615) and mixed with its curing agent. This mixture was deposited by using a prototype tool and degassed and cured at $75^{\circ} \mathrm{C}$ for $12 \mathrm{~h}$ (see figure 4). This standard PDMS has some advantages, however a number of properties inherent to PDMS limits its capabilities in the soft UV-NIL. First, the Young's modulus of standard PDMS is low and can limit the fabrication of high density patterns at a sub-100 nm scale due to collapse of structures. Second, the surface energy $(\sim 20 \mathrm{mN} / \mathrm{m})$ of PDMS is not low enough to duplicate profiles with high fidelity. To finish, the high elasticity and thermal expansion can lead to deformations and distortions during the fabrication process.

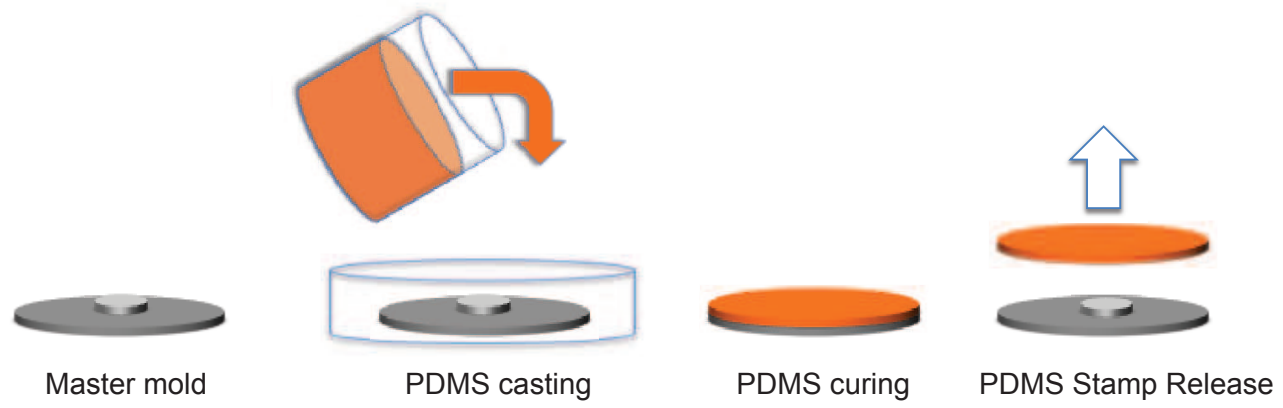

Fig. 4. Scheme of the standard process for the fabrication of the soft stamp.

To improve the resolution and fidelity of structures in the soft UV-NIL, the mechanical properties of the soft stamp need to be improved. Thus, a thin layer of hard PDMS 
(50 $\mu \mathrm{m})$ is used and supported by a standard PDMS layer (1.5 $\mathrm{mm})$ (see figure 5). This second layer allows to keep a good flexibility and adaptation on the spin coated wafer during imprint transfer (Plachetka et al., 2005). Then, the bilayer stamp is fixed on a glass carrier. The hard PDMS is a specific thermocured siloxane polymer based on copolymers Vinylmethylsiloxane-Dimethylsiloxane (VDT301) and Methyl-hydrosilane-Dimethylsiloxane (HMS-301) from ABCR, respectively, $34 \mathrm{~g}$ and $11 \mathrm{~g}$ (Choi et al., 2004). In addition, before degassing the mixture with a mixing machine we add $50 \mu \mathrm{L}$ of platinum catalyst, and $0.5 \% \mathrm{w} / \mathrm{w}$ modulator tetramethyl-tetravinyl cyclotetrasiloxane from FLUKA to the mixture (Schmid \& Michel, 2000). The hard PDMS is spin coated on the silicon master mold which has been treated with the TriMethylChloroSilane (TMCS) anti-sticking layer. The standard PDMS (RTV 615) with its curing agent are mixed before coating on the thin hard layer PDMS (H-PDMS). Then the sample is cured at $75^{\circ} \mathrm{C}$ overnight.

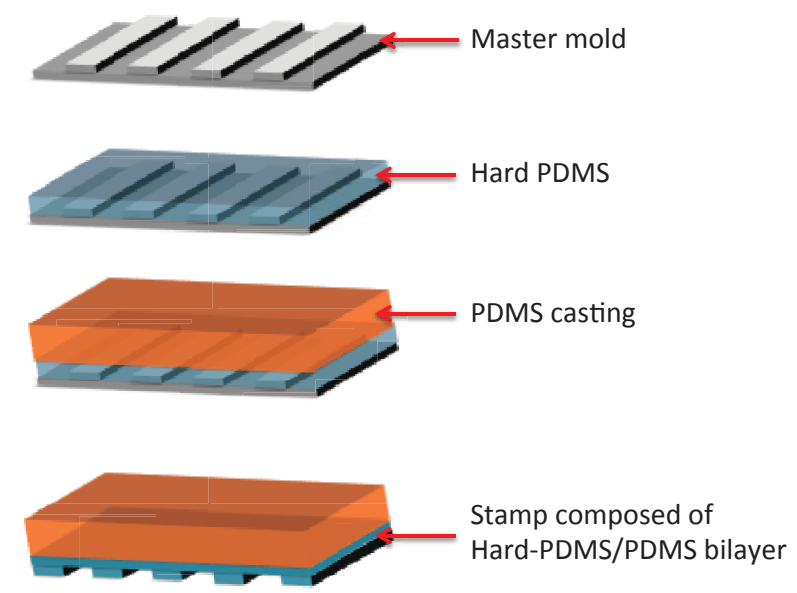

Fig. 5. Principle scheme of the fabrication process of the Hard-PDMS/PDMS stamp.

For the chosen example of nanostructures, this type of soft stamp is very suitable. Indeed, the nanodots dimensions are $80 \mathrm{~nm}$ of diameter and the periodicity of $250 \mathrm{~nm}$. The figure 6(a) represents an AFM image of the obtained H-PDMS stamp.

In addition, the SEM image of Fig.6(b) shows nanopatterned hexane diluted PDMS (at 5\%, current agent $1 / 10$ ) surface of around $100 \mu \mathrm{m}^{2}$ by using AAO templates for the fabrication of this soft stamp. The topography of the AAO molds has been well transferred in the PDMS stamp over centimetres of surface area. The stamp is composed of micro-domains of regularly organized nanobumps. Each bump is around $180 \mathrm{~nm}$ height and $250 \mathrm{~nm}$ diameter. For this fabrication, the PDMS has been simply spread on the AAO membranes without any external pressure but its own weight. Before this spreading, the standard PDMS with the curing agent and the hexane solvent are mixed and cured at $60^{\circ} \mathrm{C}$ for $12 \mathrm{~h}$. The obtained PDMS layer thickness is around $3 \mathrm{~mm}$. After the curing step, the PDMS layer is peeled off manually. An advantage of using AAO templates is their low surface reactivity compared to silicon dioxide surfaces. Thus, an anti-adhesive layer is not necessary and possible interactions with the used solvent can be avoided, when molding and de-molding PDMS from AAO templates. 

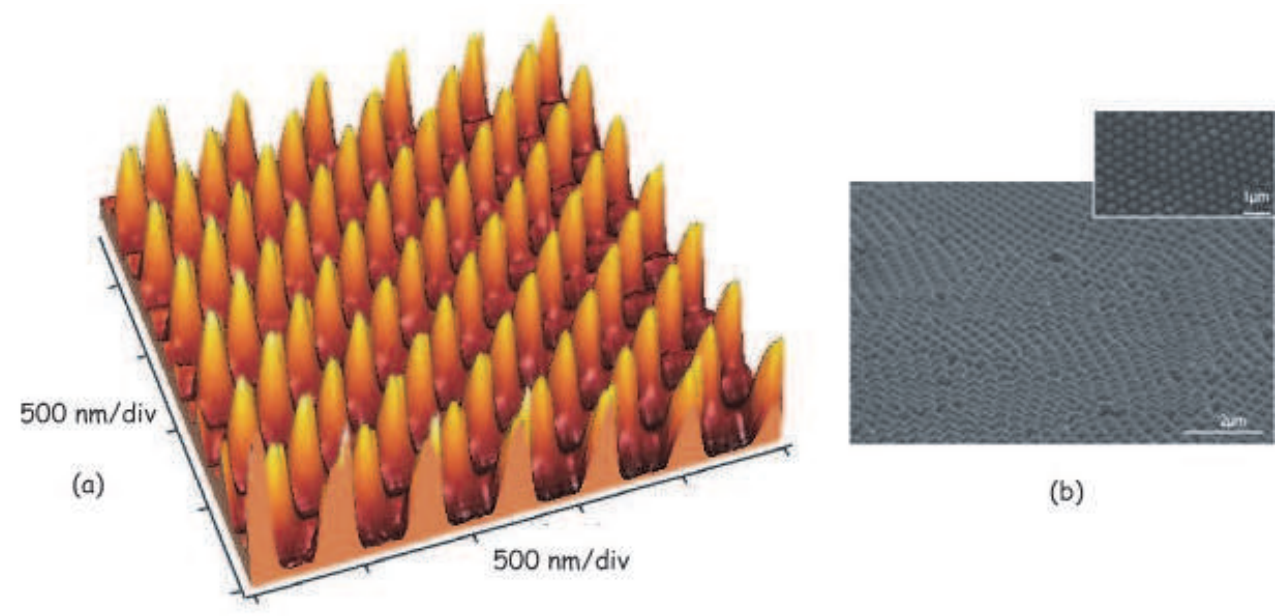

(b)

Fig. 6. (a) AFM image of the dots in H-PDMS stamp (periodicity: $\sim 250 \mathrm{~nm}$, diameter (FWHM): $\sim 80 \mathrm{~nm}$ and height: $80 \mathrm{~nm}$ ), (b) SEM image of stamp of PDMS hexane diluted at $5 \%$, tilted at $55^{\circ}$, and the insert shows a top view.

\subsection{Third step of fabrication: Soft UV-NIL in AMONIL \& gold nanodisks fabrication}

\subsubsection{Soft UV-NIL in AMONIL}

Several UV-sensitive resists exist as the NXR 2010 and the AMONIL. Both AMONIL resist and NXR 2010 resist exhibit good performance for resolution and etching resistance. The AMONIL resist was chosen for its low cost compared to the NXR 2010 resist, its excellent time of conservation and AMONIL resist is a mixture of organic and inorganic compounds having a surface energy of $39.5 \mathrm{mN} / \mathrm{m}$. AMONIL MMS4 from AMO GmbH is used and spin coated on the top of a PMMA A2 underlayer $(100 \mathrm{~nm}$ thick, surface energy $=40.2$ $\mathrm{mN} / \mathrm{m}$ ), which is the etching mask for final RIE step and which allows the AMONIL lift-off after curing. For our experiments, an AMONIL thickness of $120 \mathrm{~nm}$ is chosen. Then, the imprint process is performed in AMONIL with UV exposure at $365 \mathrm{~nm}$ wavelength with 10 $\mathrm{mW} / \mathrm{cm}^{2}$ power during exposure time of $20 \mathrm{~min}$. The pressure used to imprint is $200 \mathrm{mbar}$. All these parameters were optimized for the fabrication of nanostructures, which use the soft stamp obtained from Si master mold. The figure 7(a) represents the imprint in AMONIL. The dimensions obtained for nanoholes imprinted in AMONIL are $\sim 80 \mathrm{~nm}$ of diameter and $\sim 250$ $\mathrm{nm}$ of periodicity and these values are in good agreement with the dimensions of nanoholes of Si master mold.

Concerning the soft stamp obtained with the AAO templates, the thicknesses of the PMMA A2 underlayer and AMONIL are $130 \mathrm{~nm}$ and $150 \mathrm{~nm}$, respectively. The imprint pressure is achieved by the simple deposit of the PDMS stamp on the top AMONIL layer without any additional (or external) pressure. Only its own weight $(\sim 1 \mathrm{~g})$ ensures the imprint. For a PDMS stamp of one square centimeter, this would correspond to a pressure of around $70 \mathrm{~Pa}$ (Hamouda et al., 2010). In the figure 7(b), the topography of the AMONIL layer after molding is presented. This surface topography is very similar to the AAO master mold. During the imprint, the stamp penetrates completely in the AMONIL layer. The thickness of this layer 

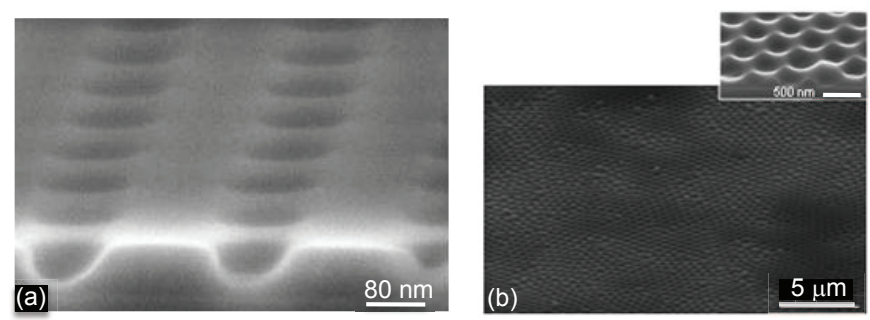

Fig. 7. SEM images of imprint in AMONIL: (a) with soft stamp obtained from Si master mold, and (b) from AAO master mold, the insert is a zoom of imprint of image (b) (scale bar $=500 \mathrm{~nm}$ ).

is weaker than the as-prepared bump heights. Thus, these results demonstrate the imprint feasibility in AMONIL using the stamps realized with the AAO templates on large zones $\left(\mathrm{cm}^{2}\right)$. However, the diameters of nanoholes obtained for the imprint in AMONIL are larger (diameter $=\sim 250 \mathrm{~nm}$ ) than those designed with the AAO master molds. This difference is due to the bad penetration of PDMS in nanoholes. We demonstrated that the penetration is better when the PDMS is well-diluted with hexane (Hamouda et al., 2011). We must still improve the fabrication process of soft stamps.

\subsubsection{Gold nanodisks fabrication}

Before the fabrication of gold nanodisks, the residual AMONIL thickness in the ground of the nanoholes needs a suitable RIE process before the etch of the PMMA resist. Indeed, this residual thickness is around $30 \mathrm{~nm}$. For the removal of this residual layer, the etch conditions (RIE) are: a flow rate of $2 \mathrm{sccm}$ of $\mathrm{O}_{2}, 20 \mathrm{sccm}$ of $\mathrm{CHF}_{3}$, with a power of $\mathrm{P}=25 \mathrm{~W}$, a pressure of $7 \mathrm{mTorr}$ and an autopolarization voltage of $430 \mathrm{~V}$. This gives an etch rate of $18 \mathrm{~nm} / \mathrm{min}$ in AMONIL. For the removal of the PMMA A2, the conditions of RIE are: $10 \mathrm{sccm}$ of $\mathrm{O}_{2}$, a power of $10 \mathrm{~W}$, a pressure of $4.7 \mathrm{mTorr}$ and an autopolarization voltage of $280 \mathrm{~V}$. This gives an etch rate of $80 \mathrm{~nm} / \mathrm{min}$ for PMMA and $18 \mathrm{~nm} / \mathrm{min}$ for AMONIL. We have a good selectivity between the etch of PMMA and AMONIL. The next step is to evaporate a gold thin layer (50 $\mathrm{nm}$ ) in order to realize the metallic nanodisks. Previously, an adhesion layer ( $\mathrm{Cr}$ ) for gold is evaporated (3-5 nm). Then, a lift-off in acetone is used to remove the PMMA underlayer (+AMONIL) in order to obtain the gold nanodisks. All these etch, deposition and lift-off conditions are valid for the 2 types of samples. Moreover, an annealing at $250^{\circ} \mathrm{C}$ during $30 \mathrm{~min}$ for smoothing and compacting the nanodisks. Only the sample of gold nanodisks obtained from Si master mold underwent this annealing. The figure 8 presents the results obtained with the 2 methods of fabrication. We observe that the dimensions of gold nanodisks are in good agreement with the dimensions obtained with the imprint in AMONIL for the 2 methods of fabrication. Indeed, the diameters of gold nanodisks are $\sim 80 \mathrm{~nm}$ and $\sim 250$ $\mathrm{nm}$, respectively. On the SEM image 8(a), we note that the annealing has well-smoothed and compacted the gold nanodisks compared to the SEM image $8(b)$, where the gold nanodisks are not annealed.

Now, gold nanodisks will be used for the plasmonic detection of biomolecules. Only the gold nanodisks of $80 \mathrm{~nm}$ diameter will be applied to biodetection. The annealing has slightly changed the shape of gold nanodisks, but no notable change of size was observed. A weak shift is observed (1-3 nm) in the localized surface plasmon resonance (LSPR) wavelength of 

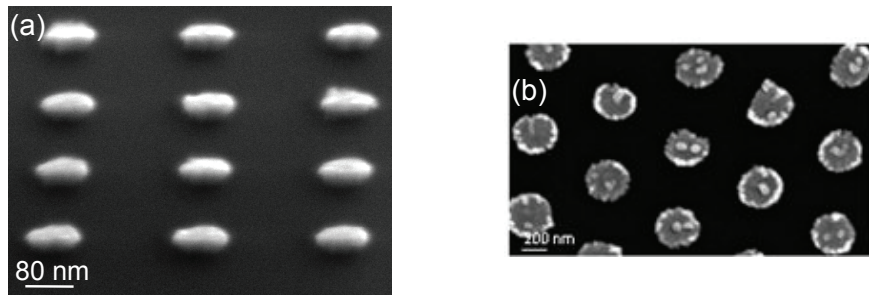

Fig. 8. SEM images of gold nanodisks with the following dimensions: (a) diameter $=\sim 80$ $\mathrm{nm}$, height $=50 \mathrm{~nm}$ and periodicity $=\sim 250 \mathrm{~nm}$, and the image tilted at $60^{\circ}$, (b) diameter $=\sim$ $250 \mathrm{~nm}$, height $=50 \mathrm{~nm}$ and the image tilted at $45^{\circ}$ (scale bar $\left.=200 \mathrm{~nm}\right)$.

the gold nanodisks arrays. However, the biomolecule detection will be not affected, because the reference will be taken on the gold nanodisks after annealing.

\section{Plasmonic detection of biomolecules}

\subsection{Adsorbate deposition}

To prepare the gold nanodisks for the detection of streptavidin (SA), which is the biomolecule that we chose for its high binding affinity $\left(\mathrm{K}_{a} \sim 10^{13} \mathrm{M}^{-1}\right)$ with the biotin molecule, the sample was biotinylated by immersion for $2 \mathrm{~h}$ in a solution $\left(1 \mathrm{mg} \cdot \mathrm{mL}^{-1}\right)$ of tri-thiolated polypeptides modified with a biotin molecule at their $\mathrm{N}$-term end and washed to remove all unbound molecules. Afterwards, we dried them with $N_{2}$ gas. Next, gold nanodisks were incubated in a given concentration of SA for 3 hours. Nanodisks were rinsed thoroughly with $10 \mathrm{mM}$ and $20 \mathrm{mM}$ PBS after biotinylation and after detection of SA to remove non-specifically bound materials. Then, we dried them with $N_{2}$ gas.

\subsection{Optical characterization of gold plasmonic nanodisks}

Visible extinction spectra of gold nanodisks were measured using a Jobin Yvon micro-Raman Spectrometer in standard transmission geometry with unpolarized white light (instrument noise level $= \pm 0.5 \mathrm{~nm}$ ). The sample is located between the white light source and the optical system of detection. The light illuminates the sample under normal incidence and the transmitted light is collected by an objective $(x 10$; N.A $=0.25)$ on a real area of $30 \times 30$ $\mu \mathrm{m}^{2}$. The extinction spectra were used to determine the position of the localized surface plasmon resonance of $\mathrm{Au}$ nanodisks and the LSPR shift of gold nanodisks after adsorption of molecules (Barbillon et al., 2007; 2008). All measurements were collected in air and to prevent atmospheric contamination, they have been performed on freshly prepared samples. The LSPR wavelength of these gold nanodisk arrays is $615 \mathrm{~nm}$ (Figure 9).

\subsection{Plasmonic sensing of streptavidin}

To validate the fabrication (UV-NIL) of these gold nanodisks and illustrate the properties of these last ones as nanosensors, we have chosen the biotin/streptavidin system. Thus, to evaluate the sensitivity of these gold nanosensors to the detection of this system, a simple model described by Campbell group is used (Jung et al., 1998):

$$
\Delta \lambda=m \Delta n\left[1-\exp \left(\frac{-2 d}{l_{d}}\right)\right]
$$


where $\Delta \lambda$ is the wavelength shift, $m$ is the refractive index sensitivity, $\Delta n$ is the change in refractive index induced by an adsorbate $\left(\Delta n=n_{\text {adsorbate }}-n_{\text {air }}\right), d$ is the effective adsorbate layer thickness and $l_{d}$ is the characteristic evanescent electric field decay length. The sensitivity $m$ does not depend on the adsorbate and according to our measurements (Barbillon et al., 2008; 2009) and those of the Van Duyne group (Jensen et al., 1999), is equal to $2 \times 10^{2}$ $\mathrm{nm}$ per refractive index unit (RIU) for our gold nanodisks arrays. To evaluate $l_{d}$, the electric field intensity is first calculated by the Finite Difference Time Domain (FDTD) method for different heights from the disk's top and then fitted according to the Prony's method using a single exponential (Barchiesi et al., 2006; Barbillon, 2010). $l_{d}$ is found equal to $14 \mathrm{~nm}$ for gold nanodisks arrays. The index difference between air and Streptavidin is $\Delta n=0.56$. The size of streptavidin is around $6 \mathrm{~nm}$ (Faure et al., 2008).

After the biotinylation, the nanodisks arrays are characterized by an LSPR wavelength at $\lambda_{L S P R}=621 \mathrm{~nm}$ (Figure 9). Figure 9 shows also that a slight redshift of $6 \mathrm{~nm}$ is observed after depositing of this biotin layer on gold nanodisks. The sample was then immersed in a PBS (Phosphate Buffered Saline) solution containing the streptavidin at a concentration equal to $1 \mathrm{nM}$ for a duration of $3 \mathrm{~h}$ and then carefully rinsed. In the figure 9 , the extinction spectrum of SA adsorption is represented and the LSPR wavelength was measured at a value of $\lambda_{L S P R}$ $=644 \mathrm{~nm}$. The real shift due to the presence of SA is then $23 \mathrm{~nm}$.

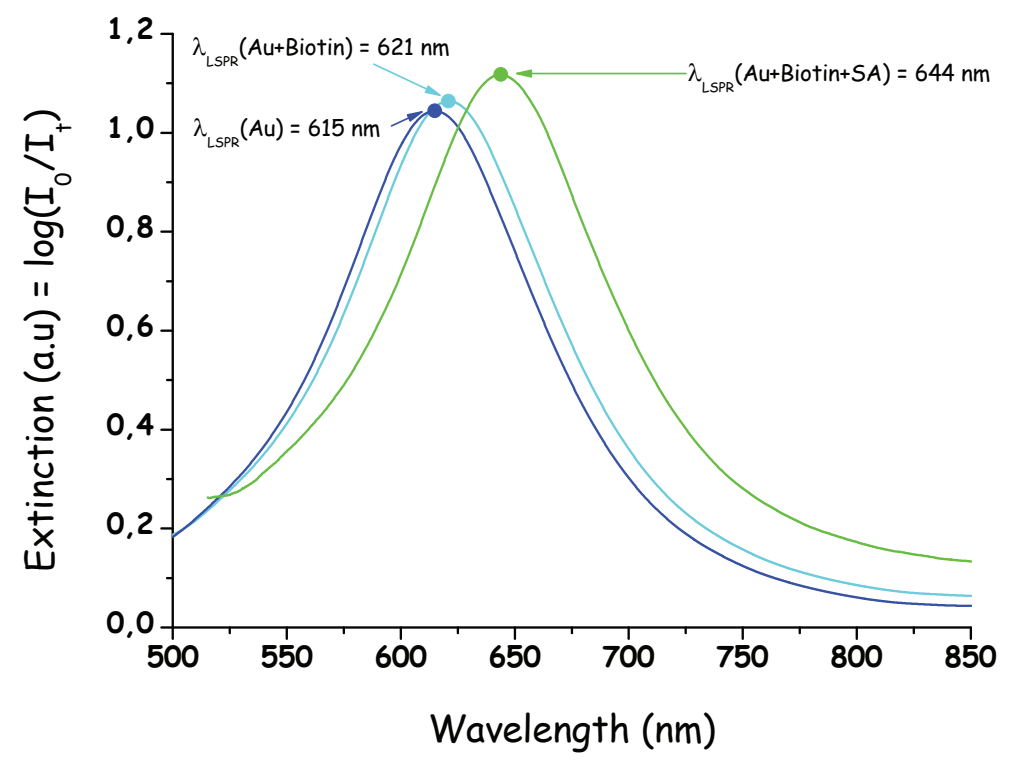

Fig. 9. Extinction spectra at each step of functionalization: in blue, Au before molecule adsorption, in cyan, Au after adsorption of biotin molecules, and in green, Au+Biotin after SA adsorption.

Knowing the following parameters: $\Delta \lambda, m, \Delta n$ and $l_{d}$, we could evaluated the value of $d$ and we found $d=1.61 \mathrm{~nm}$. From this value of $d$, the paving density can be calculated and 
a value of 0.27 was found. Compared to the maximal density obtained in the case of a hexagonal covering (0.90), the paving density of SA molecules is $30 \%$ of the maximal one for the incubation concentration used here $\left(C_{S A}=1 \mathrm{nM}\right)$.

\section{Conclusion}

In this chapter, we demonstrated that the soft UV-NIL technique could fabricated gold nanodisks on large area $\left(\mathrm{Ex}: 1 \mathrm{~mm}^{2}\right.$, and some $\left.\mathrm{cm}^{2}\right)$. The obtained dimensions of nanodisks are $80 \mathrm{~nm}$ of diameter, $250 \mathrm{~nm}$ of periodicity and $50 \mathrm{~nm}$ of height with the soft stamp designed with Si master mold. In addition, the system AAO and hexane-diluted PDMS demonstrated that it is a good candidate for UV-NIL stamp fabrication, because the advantages of using AAO are to realize inexpensive and efficient hexane-diluted PDMS stamps on large zones (some $\mathrm{cm}^{2}$ ). However, the quality of soft stamps realized with AAO must be improved in order to fabricate highly identical and well-defined nanostructures, which could be used for biological applications like bioplasmonics. A small change of the shape of Au nanodisks arrays was also observed after an annealing at $250{ }^{\circ} \mathrm{C}$ during $30 \mathrm{~min}$ which eliminates the process structural defects. A plasmonic streptavidin sensing with these gold nanodisks was studied. Finally, the gold nanodisks obtained by UV-NIL technique are very sensitive to biomolecules detection. Moreover, we could estimate the paving density of SA adsorbed on gold nanodisks. To finish, the soft UV nanoimprint lithography is very promising and relatively simple to employ for the design of plasmonic nanobiosensors.

\section{References}

Jensen, T. R.; Duval, M. L.; Kelly, K. L.; Lazarides, A. A.; Schatz, G. C. \& Van Duyne, R. P. (1999). Nanosphere Lithography : Effect of the External Dielectric Medium on the Surface Plasmon Resonance Spectrum of a Periodic Array of Silver Nanoparticles. J. Phys. Chem. B, 103, 9846-9853, ISSN 1520-5207

Barbillon, G.; Bijeon, J. -L.; Lérondel, G.; Plain, J. \& Royer, P. (2008). Detection of chemical molecules with integrated plasmonic glass nanotips. Surface Science, 602, L119-L122, ISSN 0039-6028

Faure, A. -C.; Barbillon, G.; Ou, M. G.; Ledoux, G.; Tillement, O.; Roux, S.; Fabregue, D.; Descamps, A.; Bijeon, J. -L.; Marquette, C. A.; Billotey, C.; Jamois, C.; Benyatou, T. \& Perriat, P. (2008). Core/Shell nanoparticles for multiple biological detection with enhanced sensitivity and kinetics. Nanotechnology, 19, 485103, ISSN 1361-6528

Barbillon, G.; Bijeon, J. -L.; Plain, J. \& Royer, P. (2009). Sensitive detection of biological species through localized surface plasmon resonance on gold nanodisks. Thin Solid Films, 517, 2997-3000, ISSN 0040-6090

Anker, J. N.; Lyandres, O.; Shah, N. C.; Zhao, J. \& Van Duyne, R. P. (2008). Biosensing with plasmonic nanosensors. Nat. Mater., 7, 442-453, ISSN 1476-4660

Barbillon, G.; Faure, A. -C.; El Kork, N.; Moretti, P.; Roux, S.; Tillement, O.; Ou, M. G.; Descamps, A.; Perriat, P.; Vial, A.; Bijeon, J. -L.; Marquette, C. A. \& Jacquier, B. (2008). How nanoparticles encapsulating fluorophores allow a double detection of biomolecules by localized surface plasmon resonance and luminescence. Nanotechnology, 19, 035705, ISSN 1361-6528

Krauss, P. R. \& Chou, S. Y. (1997). Nano-compact disks with $400 \mathrm{Gbit} / \mathrm{in}^{2}$ storage density fabricated using nanoimprint lithography and real with proximal probe. Appl. Phys. Lett., 71, 3174-3176, ISSN 1077-3118 
Jung, G. Y.; Johnston-Halperin, E.; Wu, W.; Yu, Z.; Wang, S. Y.; Tong, W. M.; Li, Z.; Green, J. E.; Sheriff, B. A.; Boukai, A.; Bunimovich, Y.; Heath J. R. \& Stanley Williams, R. (2006). Circuit Fabrication at 17 nm Half-Pitch by Nanoimprint Lithography. Nano Lett., 6 , 351-354, ISSN 1530-6992

Austin, M. D.; Zhang, W.; Ge, H.; Wasserman, D.; Lyon, S. A. \& Chou, S. Y. (2005). 6 nm half-pitch lines and $0.04 \mu \mathrm{m}^{2}$ static random access memory patterns by nanoimprint lithography. Nanotechnology, 16, 1058, ISSN 1361-6528

Hamouda, F.; Barbillon, G.; Held, S.; Agnus, G.; Gogol, P.; Maroutian, T.; Scheuring, S. \& Bartenlian, B. (2009). Nanoholes by soft UV nanoimprint lithography applied to study of membrane proteins. Microelectron. Eng., 86, 583-585, ISSN 0167-9317

Hamouda, F.; Barbillon, G.; Gaucher, F. \& Bartenlian, B. (2010). Sub-200 nm gap electrodes by soft UV nanoimprint lithography using polydimethylsiloxane mold without external pressure. J. Vac. Sci. Technol. B, 28, 82-85, ISSN 0003-6951

Barbillon, G.; Hamouda, F.; Held, S.; Gogol, P. \& Bartenlian, B. (2010). Gold nanoparticles by soft UV nanoimprint lithography coupled to a lift-off process for plasmonic sensing of antibodies. Microelectron. Eng., 87, 1001-1004, ISSN 0167-9317

Sengupta, K.; Moyen, E.; Macé, M.; Benoliel, A. -M.; Pierres, A.; Thibaudau, F.; Masson, L.; Limozin, L.; Bongrand, P. \& Hanbücken, M. (2009). Large-Scale Ordered Plastic Nanopillars for Quantitative Live-Cell Imaging. Small, 5, 449-453, ISSN 1613-6829

Masuda, H. \& Fukuda, K. (1995). Ordered Metal Nanohole Arrays by a Two-Step Replication of Honeycomb Structures of Anodic Alumina. Science, 268, 1466-1468, ISSN 1095-9203

Hamouda, F.; Sahaf, H.; Held, S.; Barbillon, G.; Gogol, P.; Moyen, E.; Aassime, A.; Moreau, J.; Canva, M.; Lourtioz, J. -M.; Hanbücken, M. \& Bartenlian, B. (2011). Large area nanopatterning by combined anodic aluminum oxide and soft UV-NIL technologies for applications in biology. Microelectron. Eng., doi: 10.1016/j.mee.2011.02.013, ISSN 0167-9317

Barbillon, G.; Bijeon, J. -L.; Plain, J.; Lamy De La Chapelle, M.; Adam, P. -M. \& Royer, P. (2007). Electron beam lithography designed chemical nanosensors based on localized surface plasmon resonance. Surface Science, 601, 5057-5061, ISSN 0039-6028

Barbillon, G.; Bijeon, J. -L.; Bouillard, J. -S.; Plain, J.; Lamy De La Chapelle, M.; Adam, P. -M. \& Royer, P. (2008). Detection in near-field domain of biomolecules adsorbed on a single metallic nanoparticle. Journal of Microscopy, 229, 270-274, ISSN 1365-2818

Bender, M.; Plachteka, U.; Ran, J.; Fuchs, A.; Vratzov, B.; Kurz, H.; Glinsner, T. \& Lindner, F. (2004). High resolution lithography with PDMS molds. J. Vac. Sci. Technol. B, 22, 3229-3232, ISSN 0003-6951

Hsia, K. J.; Huang, Y.; Menard, E.; Park, J. -U.; Zhou, W.; Rogers, J. \& Fulton, J. M. (2005). Collapse of stamps for soft lithography due to interfacial adhesion. Appl. Phys. Lett., 86, 154106, ISSN 0003-6951

Schmid, H.; Biebuyck, H.; Michel, B. \& Martin, O. J. M. (1998). Light-coupling masks for lensless, sub-wavelength optical lithography. Appl. Phys. Lett., 72, 2379, ISSN 0003-6951

Plachteka, U.; Bender, M.; Fuchs, A.; Vratzov, B.; Glinsner, T.; Lindner, F. \& Kurz, H. (2005). Wafer scale patterning by soft UV-Nanoimprint Lithography. Microelectron. Eng., 73/74, 167-171, ISSN 0167-9317

Choi, D. -G.; Yu, H. -K. \& Yang, S. -M. (2004). 2D nano/micro hybrid patterning using soft/block copolymer lithography. Mater. Sci. Eng. C, 24, 213-216, ISSN 0928-4931 
Schmid, H. \& Michel, B. (2000). Siloxane Polymers for High-Resolution, High-Accuracy Soft Lithography. Macromolecules, 33, 3042-3049, ISSN 1520-5835

Jung, L. -S.; Campbell, C. -T.; Chinowsky, T.-M.; Mar, M. -N. \& Yee, S. -S. (1998). Quantitative Interpretation of the Response of Surface Plasmon Resonance Sensors to Adsorbed Films. Langmuir, 14, 5636-5648, ISSN 1520-5827

Barchiesi, D.; Grosges, T. \& Vial, A. (2006). Measurement of decay lengths of evanescent waves: the lock-in nonlinear filtering. New J. Phys., 8, 263, ISSN 1367-2630

Barbillon, G. (2010). Determination of Evanescent Electric Field Decay Length of Metallic Nanodisks by Using Localized Surface Plasmon Spectroscopy. Journal of Materials Science and Engineering, 4, 69-74, ISSN 1934-8959 


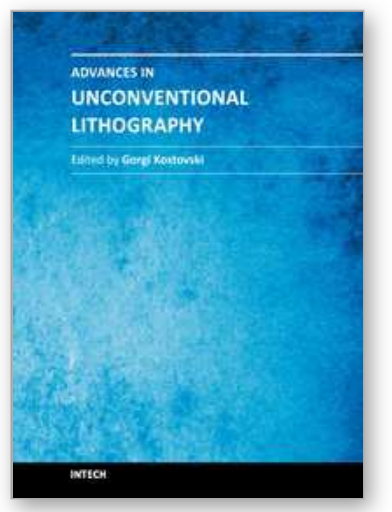

\author{
Advances in Unconventional Lithography \\ Edited by Dr. Gorgi Kostovski
}

ISBN 978-953-307-607-2

Hard cover, 186 pages

Publisher InTech

Published online 09, November, 2011

Published in print edition November, 2011

The term Lithography encompasses a range of contemporary technologies for micro and nano scale fabrication. Originally driven by the evolution of the semiconductor industry, lithography has grown from its optical origins to demonstrate increasingly fine resolution and to permeate fields as diverse as photonics and biology. Today, greater flexibility and affordability are demanded from lithography more than ever before. Diverse needs across many disciplines have produced a multitude of innovative new lithography techniques. This book, which is the final instalment in a series of three, provides a compelling overview of some of the recent advances in lithography, as recounted by the researchers themselves. Topics discussed include nanoimprinting for plasmonic biosensing, soft lithography for neurobiology and stem cell differentiation, colloidal substrates for two-tier self-assembled nanostructures, tuneable diffractive elements using photochromic polymers, and extreme-UV lithography.

\title{
How to reference
}

In order to correctly reference this scholarly work, feel free to copy and paste the following:

Grégory Barbillon (2011). Soft UV Nanoimprint Lithography: A Tool to Design Plasmonic Nanobiosensors, Advances in Unconventional Lithography, Dr. Gorgi Kostovski (Ed.), ISBN: 978-953-307-607-2, InTech, Available from: http://www.intechopen.com/books/advances-in-unconventional-lithography/soft-uv-nanoimprintlithography-a-tool-to-design-plasmonic-nanobiosensors

\section{INTECH}

open science | open minds

\section{InTech Europe}

University Campus STeP Ri

Slavka Krautzeka 83/A

51000 Rijeka, Croatia

Phone: +385 (51) 770447

Fax: +385 (51) 686166

www.intechopen.com

\section{InTech China}

Unit 405, Office Block, Hotel Equatorial Shanghai

No.65, Yan An Road (West), Shanghai, 200040, China

中国上海市延安西路65号上海国际贵都大饭店办公楼 405 单元

Phone: +86-21-62489820

Fax: $+86-21-62489821$ 
(C) 2011 The Author(s). Licensee IntechOpen. This is an open access article distributed under the terms of the Creative Commons Attribution 3.0 License, which permits unrestricted use, distribution, and reproduction in any medium, provided the original work is properly cited. 